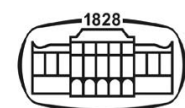

AKADÉMIAI KIADÓ

\title{
Molecular emm typing of Bulgarian
}

macrolide-resistant Streptococcus pyogenes isolates

\section{Acta Microbiologica et Immunologica Hungarica}

67 (2020) 1, 14-17

DOI: $10.1556 / 030.66 .2019 .033$

(c) 2019 Akadémiai Kiadó, Budapest

ORIGINAL ARTICLE

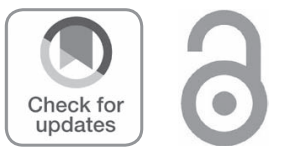

* Corresponding author: Adile Muhtarova Department of Medical Microbiology, Faculty of Medicine, Medical University of Sofia, 2 Zdrave str. 1431-Sofia, Bulgaria

Phone: +359 29172 520;

Fax: +359 29515317

E-mail: adimuhtarova@gmail.com

\author{
ADILE MUHTAROVA ${ }^{1 *}$, KALINA MIHOVA ${ }^{2}$, \\ RUMYANA MARKOVSKA ${ }^{1}$, IVAN MITOV ${ }^{1}$, \\ RADKA KANEVA ${ }^{2}$ and RAINA GERGOVA ${ }^{1}$
}

\footnotetext{
${ }^{1}$ Department of Medical Microbiology, Faculty of Medicine, Medical University of Sofia, Sofia, Bulgaria

${ }^{2}$ Department of Medical Chemistry and Biochemistry, Molecular Medicine Center, Faculty of Medicine, Medical University, Sofia, Bulgaria
}

Received: May 14, 2018 • Accepted: September 06, 2019 • Published online: December 13, 2019

\begin{abstract}
Group A streptococcus (GAS) is a human pathogen causing a broad range of infections, linked with global morbidity and mortality. Macrolide resistance rates vary significantly in different parts of the world. Driving factors of the emergence and spread of resistant clones are not clearly understood. We investigated 102 macrolide-resistant GAS strains collected during the period 2014-2018 from various clinical specimens from Bulgarian patients. Strains were characterized by the presence of $m e f A / m e f E$, ermA, and ermB using polymerase chain reaction and sequencing for $m e f A / m e f E$. Resistant strains were studied by $\mathrm{emm}$ sequence typing and emm-cluster system. Most prevalent $\mathrm{emm}$ types among the macrolide-resistant GAS strains were emm 28 (22.55\%), emm 12 (17.65\%), and emm 4 (16.66\%). Almost all (87.25\%) of the macrolide-resistant isolates harboring ermB were emm 28 . The isolates that carried ermA were predominantly emm 12 (38.24\%) and emm77 (38.24\%), with fewer emm89 (23.53\%). The isolates harbored predominantly mefE (49 isolates) and only 9 strains carried mefA. The most prevalent emm clusters among the GAS isolates were E4 (40.20\%), A-C4 (17.65\%), and E1 (16.66\%). The study's results suggest that dissemination of specific clones in GAS population may also be the reason for the increasing macrolide-resistance rate in our country.
\end{abstract}

\section{KEYWORDS}

Streptococcus pyogenes, emm types, macrolide resistance

\section{INTRODUCTION}

Group A streptococcus (GAS), or Streptococcus pyogenes, is a human pathogen responsible for a broad range of infections and is an important cause of global morbidity and mortality [1]. Penicillin has been the drug of first choice for the treatment of infections caused by this microorganism since the 1940s and is still effective [2, 3]. However, in the past few years, $20 \%-40 \%$ failure has been reported for this treatment [4]. Macrolides are important alternatives for treatment of patients who are allergic to penicillin or in cases of penicillin treatment failure [5]. The use of clindamycin for life-threatening invasive infections may reduce the mortality considerably [6]. Unfortunately, the use of these antibiotics is limited due to the increasing macrolide resistance in some countries [7-11]. A new method for understanding the epidemiology of the GAS types is emm cluster typing, based on the $\mathrm{M}$ protein, which groups $\mathrm{emm}$ types into 48 different functional emm clusters on the basis of their structural properties [12].

This study aimed to determine the emm types distribution of Bulgarian clinical GAS isolates harboring macrolide-resistance determinants. 


\section{MATERIAL AND METHODS}

A collection of 102 macrolide-resistant GAS strains was included in this study. The isolates originated from throat swabs ( $n=68)$ (with a diagnosis of tonsillopharyngitis and scarlet fever), peritonsillar abscess punctures $(n=5)$, ear punctures $(n=11)$, and wound secretion $(n=18)$, during the period 2014-2018. The age range of the patients was $3-85$ years. Strain identification and the polymerase chain reaction (PCR) protocols used for detection of the macrolide resistance genes (ermB, ermA, and $m e f A / m e f E$ ) were performed as previously described [8]. The amplified segments with primers for $m e f A / E$ were sequenced in both strands and analyzed with DNAMAN version 8.0 Software (Lynnon BioSoft, Vaudreuil-Dorion, Canada).

The genotypes were determined by sequence analysis of the variable $5^{\prime \prime}$ region of the emm gene after amplification by PCR in accordance with the protocol of the Centers for Disease Control and Prevention (https://www.cdc.gov/ streplab/groupa-strep/emm-typing-protocol.html), using the following primers $\mathrm{F}$ : GGGAATTCTATTSGCTTAGAA AATTAA, R: GCAAGTTCTTCAGCTTGTTT [13]. The amplification products were separated in a $2 \%$ agarose gel for $90 \mathrm{~min}$ at $120 \mathrm{~V}$, stained with ethidium bromide $(0.5 \mathrm{mg} / \mathrm{L})$, and detected by UV transillumination (wavelength $=312 \mathrm{~nm}$ ). The gene $\mathrm{emm}$ was sequenced using a BigDye Terminator v3.1 Cycle Sequencing Kit and BigDye Terminator v1.1 and v3.1 5X Sequencing Buffer in an Applied Biosystems 3130xl Genetic Analyzer. The data were analyzed and edited using Chromas version 2.1. The $5^{\prime}$ ends of the emm sequences were compared to sequences in the CDC database (http//:www.cdc. gov/ncidod/biotech/strep/strepblast.html) and emm genotypes were clustered according to the data in the CDC database.

\section{RESULTS}

The distribution of the emm types in 102 GAS isolates with presence of different macrolide resistance genes is presented in Table I. As shown, the most prevalent emm types among all the tested strains were emm 28 (22.55\%), emm 12 (17.65\%), and emm4 (16.66\%), accounting for over $50 \%$ of isolates. About $87.25 \%$ of the macrolide-resistant isolates harboring ermB were emm28. The isolates that carried the ermA gene were predominantly emm 12 (38.24\%) and emm 77 (38.24\%), and fewer were emm89 (23.53\%). The isolates harboring mefA/ $m e f E$ were the most heterogeneous according to the emm types (Table I). Sequencing the mef genes of our isolates revealed that 49 strains were positive for mefE, $100 \%$ identical with the corresponding segment of published sequence of mefE gene with accession number EU870854.1, and 9 strains showed presence of mefA genes ( $100 \%$ identical with published sequence accession number AF227521.1). Various emm types were presented in strains with $m e f E$ and $m e f A$, respectively, where no clonal association was found. All the emm types in this study belonged to six emm clusters. The most prevalent clusters among the Bulgarian macrolide-resistant GAS isolates were E4 (40.20\%), A-C4 (17.65\%), and E1 (16.66\%), observed in over $50 \%$ of the examined isolates.

\section{DISCUSSION}

Increasing macrolide resistance is mostly associated with increased local consumption of macrolide antibiotics or with the spread of specific macrolide-resistant clones [14, 15]. According to the ECDC, Bulgaria was the seventh largest

Table I. Distribution of emm types, emm clusters, and macrolide-resistance genes observed in examined 102 GAS strains

\begin{tabular}{|c|c|c|c|c|c|}
\hline \multirow[b]{3}{*}{ emm type } & \multirow{3}{*}{$\begin{array}{l}\text { No. of isolates } \\
{[n(\%)]}\end{array}$} & \multirow[b]{3}{*}{ emm Cluster } & \multicolumn{3}{|c|}{ Genes encoded macrolide resistance in the tested 102 isolates } \\
\hline & & & mefA/mefE & ermA & ermB \\
\hline & & & $58(56.86)$ & $19(18.63)$ & $25(24.50)$ \\
\hline emm1 & $12(11.76)$ & A-C3 & $12(20.69)$ & 0 & 0 \\
\hline emm2 & 4 (3.92) & E4 & $4(6.9)$ & 0 & 0 \\
\hline emm3 & $12(11.76)$ & A-C5 & $11(18.96)$ & 0 & $1(4)$ \\
\hline emm4 & $17(16.66)$ & E1 & $17(29.31)$ & 0 & 0 \\
\hline emm6 & $2(1.96)$ & M6 & $2(3.45)$ & 0 & 0 \\
\hline emm12 & 18 (17.65) & A-C4 & 8 (13.79) & $8(42.10)$ & $2(8)$ \\
\hline emm28 & $23(22.55)$ & E4 & 1 (1.72) & 0 & $22(88)$ \\
\hline emm77 & $8(7.84)$ & E4 & 0 & $8(42.10)$ & 0 \\
\hline \multirow[t]{2}{*}{ emm89 } & $6(5.88)$ & E4 & $3(5.17)$ & 3 (15.79) & 0 \\
\hline & $102(100)$ & & $58(100)$ & $19(100)$ & $25(100)$ \\
\hline
\end{tabular}

Note: GAS: group A streptococcus. 
consumer of macrolides, lincosamides, and streptogramins in the community and hospital sector in Europe in 2017 [16]. In parallel, the macrolide-resistance rate in Bulgaria has increased from $23 \%$ in $2013-2014$ to nearly $40 \%$ in $2015-2016$ [8]. The fluctuations in the macrolide resistance rates do not always depend on the macrolide consumption in a society [17]. It is also possible that increase in the macrolide resistance in our area is associated with the widespread of specific clones, like $\mathrm{emm} 28$ possessing the $\mathrm{ermB}$ gene. Over the past 20 years, there have been studies showing similar clonal distribution of the multiresistant emm 28 clone in other European countries, such as Spain, France, and Belgium [18-20]. The current work showed that macrolide-resistant isolates carrying the ermA gene are predominantly presented in genotypes emm77 and emm12, similar to the results from earlier Serbian studies [21]. A large-scale study showed that emm 28 and emm12 were included in the major seven identified emm types in Europe and North America, especially emm 28 in Denmark, Finland, and Germany [22]. After 2013, ermB has been reported in $22.55 \%$ of resistant strains [8] and this study showed that almost $90 \%$ of GAS-harboring ermB belonged to emm 28 . This data indicates that the clonal association is the other reason for the rise in macrolide-resistance levels in Bulgaria.

All the macrolide-resistant emm types found in this study have been included in a new 30 -valent $M$ protein-based vaccine, which is under development [23]. Recently, the emm cluster system can give new understanding and support to vaccine design and evaluation of GAS infections and their complications [12]. In this study, we identified six emm clusters among the macrolide-resistant GAS strains. The most prevalent $\mathrm{emm}$ clusters among the macrolide-resistant isolates from our area, which were E4 (40.20\%), A-C4(17.65\%), and E1(16.66\%), showed similarity with the emm cluster identified in pharyngeal and non-pharyngeal pediatric isolates from Greece [24], but not in the Pacific region [25].

\section{CONCLUSIONS}

To our knowledge, this is the first study that applies the emm cluster typing system in Bulgarian macrolide-resistant GAS isolates. We also suggest that clonal dissemination among the GAS population (like emm28 possessing the ermB gene) is responsible for the increasing macrolide-resistance rate in our country.

Acknowledgements: This study was supported by the Medical University of Sofia (Council of Medical Science, project number: 7639/20.11.2017, grant number: D-57/2018).

Conflict of Interest: The authors declare no conflict of interest.

\section{REFERENCES}

1. Tanaka, H., Katsuragi, S., Hasegawa, J., Tanaka, K., Osato, K., Nakata, M., Murakoshi, T., Sekizawa, A., Kanayama, N.,
Ishiwata, I., Ikeda, T.: The most common causative bacteria in maternal sepsis-related deaths in Japan were group A streptococcus: A nationwide survey. J Infect Chemother 25, 41-44 (2019).

2. Abraham, T., Sistla, S.: Trends in antimicrobial resistance patterns of Group A streptococci, molecular basis and implications. Indian J Med Microbiol 36, 186 (2018).

3. Cattoir, V.: Mechanisms of antibiotic resistance. In Ferretti, J. J., Stevens, D. L., Fischetti, V. A. (eds): Streptococcus pyogenes: Basic Biology to Clinical Manifestations [Internet]. University of Oklahoma Health Sciences Center, Oklahoma City, 2016.

4. Pichichero, M. E., Casey, J. R.: Systematic review of factors contributing to penicillin treatment failure in Streptococcus pyogenes pharyngitis. Otolaryngol Head Neck Surg 137, 851-857 (2007).

5. Stevens, D. L., Bisno, A. L., Chambers, H. F., Dellinger, E. P., Goldstein, E. J., Gorbach, S. L., Hirschmann, J. V., Kaplan, S. L., Montoya, J. G., Wade, J. C.: Practice guidelines for the diagnosis and management of skin and soft tissue infections: 2014 update by the Infectious Diseases Society of America. Clin Infect Dis 59, e10-e52 (2014).

6. Carapetis, J. R., Jacoby, P., Carville, K., Ang, S. J. J., Curtis, N., Andrews, R.: Effectiveness of clindamycin and intravenous immunoglobulin, and risk of disease in contacts, in invasive group A streptococcal infections. Clin Infect Dis 59, 358-365 (2014).

7. Michos, A., Koutouzi, F. I., Tsakris, A., Chatzichristou, P., Koutouzis, E. I., Daikos, G. L., Stathi, A., Syriopoulou, V. P.: Molecular analysis of Streptococcus pyogenes macrolide resistance of paediatric isolates during a 7 year period (2007-13). J Antimicrob Chemother 71, 2113-2117 (2016).

8. Muhtarova, A. A., Gergova, R. T., Mitov, I. G.: Distribution of macrolide resistance mechanisms in Bulgarian clinical isolates of Streptococcus pyogenes during the years of 2013-2016. J Glob Antimicrob Resist 10, 238-242 (2017).

9. Tanaka, Y., Gotoh, K., Teramachi, M., Ishimoto, K., Tsumura, N., Shindou, S., Yamashita, Y.: Molecular epidemiology, antimicrobial susceptibility, and characterization of macrolideresistant Streptococcus pyogenes in Japan. J Infect Chemother 22, 727-732 (2016).

10. Olivieri, R., Morandi, M., Zanchi, A., Tordini, G., Pozzi, G., De Luca, A., Montagnani, F.: Evolution of macrolide resistance in Streptococcus pyogenes over 14 years in an area of central Italy. Indian J Med Microbiol 64, 1186-1195 (2015).

11. Lu, B., Fang, Y., Fan, Y., Chen, X., Wang, J., Zeng, J., Li, Y., Zhang, Z., Huang, L., Li, H., Li, D., Zhu, F., Cui, Y., Wang, D.: High prevalence of macrolide-resistance and molecular characterization of Streptococcus pyogenes isolates circulating in China from 2009 to 2016. Front Microbiol 8, 1052 (2017).

12. Sanderson-Smith, M., De Oliveira, D. M., Guglielmini, J., McMillan, D. J., Vu, T., Holien, J. K., Curtis, N., Beall, B., Walker, M., Parker, M., Carapetis, J., Melderen, L., Sriprakash, K., Smeesters, P.: A systematic and functional classification of Streptococcus pyogenes that serves as a new tool for molecular typing and vaccine development. J Infect Dis 210, 1325-1338 (2014).

13. Green, N. M., Beres, S. B., Graviss, E. A., Allison, J. E., McGeer, A. J., Vuopio-Varkila, J., LeFebre, R., Musser, J. M.: Genetic 
diversity among type emm 28 group A streptococcus strains causing invasive infections and pharyngitis. J Clin Microbiol 43, 4083-4091 (2005).

14. McGowan, J. E., Jr.: Antimicrobial resistance in hospital organisms and its relation to antibiotic use. Rev Infect Dis 5, 1033-1048 (1983).

15. Kaplan, E. L.: Recent evaluation of antimicrobial resistance in $\beta$-hemolytic streptococci. Clin Infect Dis 24, S89-S92 (1997).

16. ESAC: Antimicrobial Consumption Database (ESAC-Net). Available at https:/ecdc.europa.eu/en/antimicrobial-consumption/ surveillance-and-disease-data/database. Accessed on: January 1, 2019.

17. Silva-Costa, C., Friaes, A., Ramirez, M., Melo-Cristino, J.: Macrolide-resistant Streptococcus pyogenes: Prevalence and treatment strategies. Expert Rev Anti Infect Ther 13, 615-628 (2015).

18. Perez-Trallero, E., Garcia, C., Orden, B., Marimon, J. M., Montes, M.: Dissemination of emm 28 erythromycin-, clindamycin-and bacitracin-resistant Streptococcus pyogenes in Spain. Eur J Clin Microbiol Infect Dis 23, 123-126 (2004).

19. Mihaila-Amrouche, L., Bouvet, A., Loubinoux, J.: Clonal spread of emm type 28 isolates of Streptococcus pyogenes that are multiresistant to antibiotics. J Clin Microbiol 42, 3844-3846 (2004).

20. Malhotra-Kumar, S., Wang, S., Lammens, C., Chapelle, S., Goossens, H.: Bacitracin-resistant clone of Streptococcus pyogenes isolated from pharyngitis patients in Belgium. J Clin Microbiol 41, 5282-5284 (2003).

21. Gajic, I., Mijac, V., Ranin, L., Grego, E., Kekic, D., Jegorovic, B., Smitran, A., Popovic, S., Opavski, N.: Changes in macrolide resistance among group A streptococci in Serbia and clonal evolution of resistant isolates. Microb Drug Resist 24, 1326-1332 (2018).

22. Gherardi, G., Vitali, L. A., Creti, R.: Prevalent emm types among invasive GAS in Europe and North America since year 2000. Front Public Health 6, 59 (2018).

23. Dale, J. B., Penfound, T. A., Chiang, E. Y., Walton, W. J.: New 30-valent $M$ protein-based vaccine evokes cross-opsonic antibodies against non-vaccine serotypes of group A streptococci. Vaccine 29, 8175-8178 (2011).

24. Koutouzi, F., Tsakris, A., Chatzichristou, P., Koutouzis, E., Daikos, G. L., Kirikou, E., Syriopoulou, V., Michos, A.: Streptococcus pyogenes emm types and clusters during a 7 -year period (2007 to 2013) in pharyngeal and nonpharyngeal pediatric isolates. J Clin Microbiol 53, 2015-2021 (2015).

25. Baroux, N., D’Ortenzio, E., Amédéo, N., Baker, C., Ali Alsuwayyid, B., Dupont-Rouzeyrol, M., O’Connor, O., Steer, A., Smeesters, P. R.: The emm-cluster typing system for group A streptococcus identifies epidemiologic similarities across the Pacific region. Clin Infect Dis 59, e84-e92 (2014). 